\title{
\& Research Square

\section{Factors Related To Implementation of An Interprofessional Communication Concept In Thoracic Oncology: A Mixed-Methods Study}

Katja Krug ( $\sim$ katja.krug@med.uni-heidelberg.de )

Heidelberg University Hospital

Jasmin Bossert

Heidelberg University Hospital

Sophia Möllinger

Heidelberg University Hospital

Nicole Deis

Heidelberg University Hospital

Laura Unsöld

Heidelberg University Hospital

Anja Siegle

Heidelberg University Hospital

Matthias Villalobos

Heidelberg University Hospital

Laura Hagelskamp

Heidelberg University Hospital

Corinna Jung

Heidelberg University Hospital

Michael Thomas

Heidelberg University Hospital

Michel Wensing

Heidelberg University Hospital

\section{Research Article}

Keywords: interprofessional collaboration, attitude, communication, advanced cancer, implementation

Posted Date: January 11th, 2022

DOI: https://doi.org/10.21203/rs.3.rs-1193037/v1 
License: (c) (i) This work is licensed under a Creative Commons Attribution 4.0 International License. Read Full License 


\section{Abstract}

Background: An innovative patient-centred interprofessional communication concept with advanced lung cancer patients (Heidelberg Milestone Communication Approach, MCA) has been developed and implemented. Role changes and interprofessional communication are challenging in a busy outpatient oncology service. The aim of the study was to assess team-related factors associated with its implementation and thoracic oncology teams' attitudes towards interprofessional collaboration.

Methods: Interviews and focus groups on implementation and interprofessional collaboration in the context of MCA were conducted with healthcare staff. The topics were analysed deductively, guided by the Professional Interactions factor of the Tailored Implementation for Chronic Diseases (TICD) framework. In addition, 3 of the 4 subscales of the validated German translation of the University of the West of England Interprofessional Questionnaire (UWE-IP-D) were collected prior to implementation of MCA (t0) with follow-up data collections at 4 months (t1), 10 months (t2) and 17 months (t3). Descriptive analysis included calculating subscale sum scores and categorizing each subscale into positive, neutral and negative attitudes.

Results: Fifteen staff members participated in the interviews and focus groups. The main interprofessional interaction factors associated with implementation concerned the knowledge of the MCA and the impact of the intervention on team roles, on information sharing and on transfer processes between wards. The survey with 87 staff (44 nurses, 13 physicians, 12 psycho-social staff, 7 therapists, and 11 others) participating at least once (t0: $n=20, t 1: n=48, t 2: n=33, t 3: n=25)$ found heterogeneous attitudes. A positive attitude to 'Communication and Teamwork' was indicated by individual sum scores between 10 and $23(M=17.7, S D=3.0)$. The majority of the respondents $(n=48)$ had neutral attitudes to 'Interprofessional Interaction' with individual sum scores varying between 13 and 45 (positive attitude $n=14$, negative attitude $n=22 ; M=28.5, S D=5.6$ ). 'Interprofessional Relationships' were characterized by primarily positive attitudes $(n=80)$ (neutral: $n=6$, negative attitude $n=1 ; M=16.0, S D=3.6)$. There were no differences between collection times.

Conclusions: Positive experiences and potential shortfalls in the implementation were observed. Future introductions of interprofessional communication concepts require further activities which should address the attitudes of healthcare professionals towards interprofessional care.

Trial registration: DRKS00013469 / Date of registration: 22/12/2017.

\section{Background}

In spite of improved diagnostic and treatment options, lung cancer is associated with a limited prognosis as the diagnosis is often made at an advanced stage [1]. In light of this limited prognosis, cancer care poses major challenges for patients and informal caregivers as well as for healthcare staff due to the need to organise and prioritise different stages during the course of the disease. Effective interprofessional collaboration may help to optimise cancer care overtime. Interprofessional collaboration 
may contribute to patient-centred care and help to enhance end-of-life outcomes, such as quality of life and the amelioration of symptoms [2]. Other than benefits for patients, healthcare professionals (physicians, nurses, therapists) may also benefit from successful interprofessional collaboration through improved working climate and better workflow. Studies indicate that interprofessional collaboration enhances the understanding of key roles and responsibilities of other professions and leads to improved mutual appreciation [3].

Effective interprofessional collaboration can improve outcomes for patients with advanced cancer and professionals. However, barriers exist in clinical practice. Poor communication between different professionals is one of the greatest barriers, caused for example by professional silos. Other reasons for ineffective communication and collaboration are workforce shortages and high staff turnover in the teams. This results in difficulties in terms of continuity of care and barriers to forming a team identity and developing common mental models and trust [4].

In order to actively promote continuity in communication over the disease trajectory of advanced lung cancer patients in a German thoracic oncology service, an interprofessional patient-centred communication approach (Heidelberg Milestone Communication Approach, MCA [5]) has been developed. This project aimed to improve patient care outcomes (i.e. information needs, quality of life, mood) using structured communication with advanced lung cancer patients, while strengthening collaboration between physicians and nurses. As part of the novel approach, specially trained physicians and nurse navigators communicate together as a tandem with patients (and family caregivers) at four defined stages in the illness trajectory in so-called milestone conversations (MCs). Nurse navigators additionally contact patients between clinic appointments to follow-up on questions after appointments, symptoms and well-being. A shared documentation was introduced: Encounters with patients were documented in a shared patient file (color-coded for nurse navigators and physicians) to which other healthcare professionals on the ward also had access. Improvements in patient outcomes (addressed information needs of patients) through the intervention could be shown [6].

The training within the MCA involved physicians and nurse navigators who worked together in tandem with the patients. They were embedded in a hospital where other healthcare professionals were directly or indirectly affected by the intervention. Although other professions were not an explicit part in the concept, changes in the team work of physicians and nurse navigators may have influenced interprofessional collaboration with other members of the workforce, i.e. therapists who also have a strong relationship with the patients [5]. They met patients with whom therapeutic decisions were made in MCs. Nurses other than the nurse navigators were in contact with patients at admission, on the ward and during therapy. In addition to the trained resident physicians, physicians in rotation also conduct MCs in tandem with a trained nurse navigator without being trained themselves. Medical assistants have to arrange the schedule. Thus, the MCA not only addresses collaboration between trained physicians and nurse navigators in the tandem, but also fostered a community of practice [7] among members of the extended healthcare team. 
The present study aims to present the team-related factors associated with implementation of MCA and the attitudes to interprofessional collaboration of professions in thoracic oncology.

\section{Methods}

\section{Study design}

This study is part of a larger study using mixed-methods to explore the effects of the MCA on interprofessional collaboration of professions in thoracic oncology. Therefore, individual interviews and focus group discussions using a semi-structured interview guide were performed with staff, including nursing, psycho-social staff, diagnostic staff, administration, other therapists and physicians.

Furthermore, staff were asked to complete the validated German translation of the University of the West of England Interprofessional Questionnaire (UWE-IP-D [8]).

\section{Setting}

The study was conducted at the Department of Thoracic Oncology at the University Hospital Heidelberg, Germany. This hospital is a comprehensive cancer centre with a large catchment area and focuses on thoracic diseases, including lung cancer.

\section{Participants}

For participation in interviews or focus group discussions, a consecutive sample of healthcare staff were individually invited. Only healthcare staff providing clinical care for patients were included (physicians, nurses, therapists). Administrative staff were excluded. All participants gave their written informed consent for study participation.

All 120 members of the medical, nursing, administrative, psycho-social, pastoral care, diagnostic and therapeutic professions were included in the survey at the Department of Thoracic Oncology, University Hospital Heidelberg. They received oral and written information including background information on the project, details on participating in the survey and a copy of the questionnaire to fill in.

\section{Data Collection Tools}

The semi-structured interview guide addressed the contact to and experiences with MCA. To ease the flow of thoughts and the discussion, participants were first asked to arrange paper shapes representing themselves, other staff and the department (i.e. different wards) on a table, and position themselves within the department and in relation to other staff. Following this activity, the semi-structured interview was undertaken explaining their experiences. 
In the survey, the validated German translation of the University of the West of England Interprofessional Questionnaire (UWE-IP-D [8-10]) was used. UWE-IP-D is a self-report instrument consisting of 34 items in a set of four scales addressing different themes. Three of the 4 subscales of the UWE-IP-D, i.e.

Communication and Teamwork, Interprofessional Interaction, and Interprofessional Relationships, were used. Communication and Teamwork items are measured on a 4-point Likert scale ( 1 strongly agree, 2 agree, 3 disagree, and 4 strongly disagree) leading to sum scores between 9 and 36, with scores 9-20, 2125 , and 26-36, respectively indicating a positive, neutral, or negative self-assessment of communication and teamwork skills. Interprofessional Interaction and Interprofessional Relationship items are assessed on a 5-point Likert scale (1 strongly agree, 2 agree, 3 undecided, 4 disagree, and 5 strongly disagree). The subscale Interprofessional Interaction takes sum scores between 9 and 45, with scores 9-22, 23-31, and $32-45$, respectively, indicating positive, neutral, and negative perceptions of interprofessional interaction. Sum scores on the subscale Interprofessional Relationships vary between 8 and 40, with scores 8-20, 2127 , and 28-40, respectively, indicating positive, neutral, and negative attitudes towards the respondent's own interprofessional relationships [8]. Additionally, healthcare professionals reported gender and profession (nursing, medical, psycho-social, therapeutic, administrative, or other allied healthcare profession).

\section{Data Collection Process}

Qualitative data were collected between September 2018 and April 2019. For organisational reasons, staff were free to participate in one of the group interviews or to make an appointment for an individual interview. Both group and individual interviews were conducted face-to-face using the semi-structured interview guide in a separate and quiet room on the ward by a health-care researcher (JB). The interview guide was developed based on a literature review and in accordance with the objectives of the MCA project. Focus group and Interview questions were oriented towards eliciting open-ended responses to acquire specific information on interprofessional collaboration. The interview guide was pre-tested with one nurse to ensure that all questions were comprehensible. All interviews were digitally recorded and transcribed verbatim. The transcripts were compared with the digital recordings to correct any inaccuracies. Data were collected until saturation was reached.

Questionnaire data were collected prior to implementation of MCA (t0) in December 2017 with follow-up data collections at 4 months directly after the implementation phase (t1), 10 months (t2) and 17 months ( $\mathrm{t} 3)$.

\section{Data analysis}

Qualitative data were analysed according to Qualitative Content Analysis [11] and to the team-related determinant "Professional Interaction" on the Tailored Implementation in Chronic Diseases (TICD) Checklist [12] as a framework with a focus on organizational aspects to structure collected data into themes and sub-themes. The TICD checklist was adapted to cover the views of non-targeted (untrained) 
professionals (instead of targeted professionals only) and the influence on the implementation. Within this approach, a summary of the content was carried out by two female researchers with a background in health services research (M. Sc.) and nursing (JB, SM) deleting all expletives and repetitions. Then, the material was coded line-by-line deductively with an a priori developed system of themes derived from the interview guide and the adapted TICD checklist (Communication and influence, Team processes, Referral processes) as well as inductively with additional content emerging from the interviews. All interviews were analysed applying this approach by both data analysts to enhance concordance of coding. The analyses were compared and the coded themes were modified where required. Moreover, all interviews were intensely discussed by the two data analysts in order to ensure agreement. All qualitative data were managed and analysed using MAXQDA 12 (VERBI Software GmbH, Berlin). Quotes presented as examples in this article have been translated from German into English with due diligence and where necessary with slight adaptations to maintain meaning.

Descriptive analysis of the quantitative data included calculating subscale sum scores and categorizing each subscale into positive, neutral and negative attitudes [9]. Sum scores are presented as mean with standard deviation overall and across the assessments and categories in absolute and relative frequencies.

\section{Ethics Statement}

The study was approved by the Ethics Committee of the University Hospital Heidelberg, Germany (protocol no. S-561/2017). The trial was registered on 22/12/2017 (trial registration no. DRKS00013469).

\section{Results}

Qualitative data

Qualitative interviews with 15 staff (3 physicians, 11 nurses, 1 therapist) were conducted, which included 4 individual interviews (physicians, therapist) and 5 group interviews with 2-3 participants (nurses) each. The mean duration of the qualitative surveys was 26 min (range $9 \mathrm{~min}$ to $38 \mathrm{~min}$ ). Regarding factors associated with implementation, the participants addressed the TICD interdependent main themes regarding interprofessional collaboration: (1) Communication and influence, (2) Team processes, and (3) Referral processes.

\section{Theme 1: Communication And Influence}

The theme "Communication and influence" describes the extent to which the support of the intervention is influenced by professional opinions and communication (adapted from [12]). Related to the MCA, this theme comprises "Knowledge about the MCA", "Role of patients", and "Own role in team". 
Each of the staff interviewed had already heard of the MCA. Their knowledge in detail depended on the contact they had with the project and its participants: some staff were more informed by being part of the project, others knew it from a distance without being directly involved. In this context it was also mentioned that it would be helpful to know which people were involved in the project.

"I can't remember someone saying, we have an MCA conversation today. Never heard that, I don't know if that happens. See, so little do I know about it." (interview 1, therapist)

Role of patients

Patients acted as deliverers of information between the MCA team and other staff. The topic "Role of patients" contains statements about the milestone conversations (MCs) that staff had been informed of by patients.

The majority of interviewees working on the ward mentioned that they could not identify the patients participating in the MCA, although there were no reports of asking patients directly if they were cared for with the MCA, either. While some staff received information from their patients about their participation in the MCA, other patients never mentioned it. Patients who mentioned the MCA to the interviewees reported positive experiences and an enhancement of the treatment process.

"I have heard from patients that the contact to the MCA team is appreciated and maintained [...]. And I always find that in itself [...] an enrichment." (interview 2, physician)

Own role in team

Even if the interviewees had not received the MCA training, they were able to report how the intervention influenced the roles they had in the team. Physicians for whom MCA was a new experience sometimes felt overruled in their therapeutic decisions when working together with a (trained) nurse navigator.

"I remember a situation with a patient who did not feel well, and then the nurse had already talked to her about discontinuing therapy. That was, for me in that moment it was a bit outside their scope." (interview 3 , physician)

In addition, therapeutic conversations conducted together with a patient were perceived as physiciandominated with the nurse navigator in a secondary role.

"There were conversations which were physician-centred and the nurses had a passive role, but they contacted the patient afterwards. So there was less direct participation during the conversations" (interview 2, physician)

Participant nurses perceived evolution in the doctor-nurse relationship distinct from MCA. From their point of view, the therapeutic conversations generally were conducted in partnership and not physiciandominated. 
"There are the somewhat older physicians who are perhaps used to talking and the nurse listening, and today it is already the case with many of them that the conversation is held together. The physician and the nurse" (focus group 1, nurse 2)

Tasks and roles of the different team members were not explicitly defined in the project. Team members could flexibly adapt their roles and responsibilities as part of the implementation process. Over time, clearer definitions of tasks and roles emerged and led to a higher degree of acceptance of shifted responsibilities.

"That was at the beginning when the role of the MCA team wasn't exactly clear, that was just perhaps a little hyperactivity. Otherwise there is not much to criticise." (interview 3 , physician)

"But that was only at the beginning, I think it was clear that this was also a new concept; both for the doctors and for the nurses - in this form and therefore, that is also quite normal that one has, I say, the one or other point where one is not in agreement and that is why there is this project, I think. We have developed further, I think. Yes." (interview 4, physician)

\section{Theme 2: Team Processes}

The theme "Team processes" describes the extent to which teams are involved to support implementation (adapted from [12]). Related to the MCA, this theme comprises Team competencies, Imparting information and Barriers.

Team competencies

Team competencies include aspects and areas of competence that belong to interprofessional collaboration associated with the MCA. Therefore, only staff who were actually in direct contact to the MCA could reflect their experiences first-hand. In our sample, this concerned physicians only. Due to rotation within the hospital, not all of them were trained in MCA but sometimes conducted therapeutic patient conversations in a tandem with a trained nurse navigator.

With the introduction of the MCA, perception of physicians and nurse navigators in the tandem differed about how and when to deliver information to the patients, especially about the prognosis. From the physician's point of view, nurse navigators wanted the patients to be fully informed from the beginning, while physicians provided information in small pieces for each patient individually. Over time, the tandems found a way to lead satisfactory conversations.

"You have to be very careful and I think that nurses even expect you to explain everything at the beginning. And there were some conflicts, I think with the time they (nurses in the tandem) have also seen that this works differently. Always, so to speak, individually, depending on how much the patient can accept at all, how much information they need and that's what we tend to do at the moment. Yes, I believe that the nurses are also quite content at the moment." (interview 4, physician) 
Members of a tandem perceived each other as supportive in providing information, preparing patients for the conversations, conducting and debriefing the conversations, and documentation. The collaboration within the interprofessional tandem was evaluated positively. Feedback after the MCs was appreciated.

"There was another pair of ears and debriefing the conversation I considered helpful. That what was documented, what was fed back or what kind of problem remained to be addressed at the next appointment." (interview 2, physician)

Other team members were also perceived as providing emotional support.

"Especially in critical situations, you don't have to carry the load of telling of progression or saying you cannot do any more (tumor centered therapy), you don't have to carry that alone. That should not be underestimated. So, I think this is also a moral support for the physicians, if there is someone else and supporting the people." (interview 3 , physician)

Collaboration within the team led to more knowledge about the patient: the team regularly exchanged information about the situation and clinical status of the patient. Ambiguities were timely clarified.

"Knowledge gain after the conversation, that MCA team members reflected information back to me, of the conversations and later also in the documentation which supplemented my own picture." (interview 2, physician)

MCA provided an agenda for topics to be addressed in a conversation. Although structured, the conversations sometimes took unintended turns and therefore lasted longer than planned. Still, it was considered an advantage when nurse navigators addressed patient-relevant issues during the conversation, debriefed with the patients and answered questions arising after the scheduled conversation. From the physicians' point of view, the nurse navigators tended to see the support needs of the patients during the MCs. Nurse navigators were an additional support, which helped to interpret the patient's statements and to address important issues again at a later time.

"During the conversation, there is always one or another issue, that the nurses notice and address. I think that's great [...] there are just questions they then can clarify outside (after the conversation). I consider this very good for patients because we are aware that patients receive a lot of information [...] and of course coordinating appointments keeping deadlines, medication, that is very complicated and for them (patients) very supportive. I also had challenges at the beginning but now it works well and both physicians and patients benefit very much." (interview 4, physician)

Physicians became more aware of the support provided by nurse navigators and used it more often. For patients who were perceived as needing more support, the nurse navigator was informed and able to schedule additional time for debriefing with the patients.

"We constantly call the nurses, 'do you have time, there is a patient who maybe needs more support'. I think it's very important because, in the end, we as physicians manage maybe half an hour to three 
quarter per patient, and you don't manage, depending on how much information the patient wants, to talk about everything. There are always some minor issues patients don't dare to ask the physician directly. Such questions are clarified with the nurses" (interview 4, physician)

Although the participating staff perceived advantages in bringing different professional perspectives together and acknowledged the problem-solving opportunities this offered, staff not involved in the tandem highlighted difficulties they experienced figuring out how to include MCA into their daily routine.

"I can imagine that it (the MCA) has advantages, because everybody is sharing their view on the patient, and how problems can be solved, for the patient but also within the team maybe." (interview 2, physician)

"Well, there are some of our colleagues who cannot yet integrate MCA into our daily activities. [...]

Therefore I believe that MCA is a real support for the patient, but it is not yet a benefit for us." (focus group 1 , nurse 1)

Some interviewees reported no change in interprofessional teamwork from the introduction of the MCA. But they stressed the point of having had a good collaboration beforehand.

"Here it (interprofessional teamwork) is very good anyway. Otherwise it wouldn't work. It is trusting and very good. Still. I would be lying if I'd say this has all become much better. It is just good." (interview 3 , physician)

Imparting information

Means by which information was passed to other members of the staff, had room for improvement in the transparency of the MCA. From the interviews, it emerged that staff other than the nurse navigators and physicians who were trained and/or conducted milestone conversations (MCs) were not informed about details of the MCA project. Nevertheless, the project and the exchange with the MCA team provided additional (unstructured) information to the wards.

"It happens from time to time that an MCA-nurse comes and says: '(Name of the patient) is not well. I have already called them once, they come (here), they have this and that.' That's information we usually don't get." (Individual interview 3, physician)

To strengthen information exchange, interviewees articulated the wish to receive a summary of the MCs to improve patient support. As part of the project, the nursing staff on the ward had access to all written information on MCs. Still, they expressed the wish to have a brief oral explanation of what was addressed in the MCs and what the patient's needs were. With this information, they could continue and improve the patient's care.

"A brief feedback session if they are coming to the ward to see some patients anyway." (focus group 2, nurse 3) 
"Everything is documented. That's new, that it is in green now. And on top, it says in big letters 'MCA patient', so you know, 'I can call someone if there is something where I cannot answer or don't have time.." (focus group 5, nurse)

\section{Barriers}

Changes in interprofessional collaboration initiated by the MCA can be best understood by looking at logistic influences on and by the MCA. At the beginning of the implementation, some of the MCs did not proceed as planned. Both organizational and interpersonal factors were identified as reasons for this. In particular, the lack of a fixed place for follow-up calls or debriefing with the patient after an MC was mentioned in the area of organizational aspects.

"Because of space, that it is just a problem [...] we already say 'the admission it is very full, so please make sure that you don't talk to the patient, even if it is only for a short time, because there are already two sitting (patients) there' and we also talk on the phone, so that nobody just takes a seat, that's too much." (focus group 5, nurse 2)

In terms of interpersonal aspects, a barrier was seen at the beginning of the implementation process in that the distribution of roles and the interaction within the tandem was not yet defined. A lack of clarity regarding roles and interaction led to some insecurity on the part of physicians and nurses, which can affect the quality of the counseling. The exchange of information was not only dependent on the individual, but also on surrounding conditions, such as time. Some staff indicated that a good exchange about the patient should take place during the afternoon shift, as many time-consuming nursing and therapeutic measures were carried out on the morning shift.

"in the morning shift it is bad, there is of course little time, but in the afternoon shift I find it suitable" (focus group 3 , nurse 1)

\section{Theme 3: Referral Processes}

The theme "Referral processes" describes processes of transferring patients within and between outpatient departments and inpatient wards and interprofessional communication (adapted from [12]). Within the MCA, this theme focused on Cross-sectoral communication.

Cross-sectoral communication

Cross-sectoral communication refers to the communication between the outpatient department and the inpatient wards. Overall, staff stated that effective communication between the wards and the outpatient department played an important role for them, as it enabled them to optimize patient care and to provide it in a targeted manner. Nevertheless, communication in the context of the MCA was perceived as controversial. The exchange about patients depended on the relationship between individual members of the staff. Advantages were particularly observed when the nurse navigator was both part of the 
interprofessional tandem and additionally worked part time on the ward. Nurse navigators in the MCA who had good relationships with staff on the ward communicated more about patients' individual situations.

"I can say that it is sometimes depending on the person, the nurse who has worked in our ward, for example, I am more in contact with her because I interact with her directly [...]." (focus group 1, nurse 2)

One interviewee stated that the tight schedule on the wards allowed only narrow time frames for exchanging information, which often affected communication. The interviewee also described their reluctance to talk to someone if they felt that the other person did not have the time.

"There's bustle, pressure on from care requirements, I'd say, especially on the ward [name of the ward], so that I'm already very reluctant to approach the doctors individually." (interview 1, therapist)

Quantitative data

Of all 120 members of the staff, 87 (72,5\%; 62 female, 22 male, 3 unknown; 44 nurses, 12 psycho-social staff, 1 diagnostic staff, 4 administration, 7 therapists, 13 physicians, 1 other, 5 unknown) completed the survey at least once (t0: $n=20, t 1: n=48, t 2: n=33, t 3: n=25)$.

Attitudes towards communication and teamwork were primarily positive (mean sum score $=17.7, \mathrm{SD}=3.0$, min-max: 10-23; positive: $n=71,81.6 \%$, neutral: $n=15,17.2 \%$, negative: $n=0,0 \%)$. Attitudes did not change over time (Table 1).

The majority of the respondents showed neutral attitudes towards interprofessional interaction (mean sum score $=28.5, S D=5.7$, min-max: 13-45; positive: $n=14,16.1 \%$, neutral: $n=48,55.2 \%$, negative: $n=22$, $25.3 \%$ ). There were no changes in attitudes across assessments (Table 1).

'Interprofessional Relationships' were characterized by primarily positive attitudes overall and across assessments (Table 1 ; overall mean sum score $=16.0, S D=3.6$, min-max: $8-29$; positive: $n=80,92.0 \%$, neutral: $n=6,6.9 \%$, negative: $n=1,1.1 \%)$. 
Table 1

Attitudes of staff towards communication and teamwork, interprofessional interaction, and interprofessional relationships

\begin{tabular}{|c|c|c|c|c|c|}
\hline & & to & t1 & t2 & t3 \\
\hline \multirow{6}{*}{$\begin{array}{l}\text { Communication and } \\
\text { Teamwork }\end{array}$} & $\mathrm{n}$ & 19 & 46 & 32 & 25 \\
\hline & $M(S D)$ & $\begin{array}{l}16.7 \\
(2.3)\end{array}$ & $\begin{array}{l}17.7 \\
(3.1)\end{array}$ & $17.4(3.4)$ & $17.4(3.4)$ \\
\hline & $\min _{36)}-\max (9-$ & $13-20$ & $10-23$ & $11-24$ & $10-24$ \\
\hline & positive $\mathrm{n}(\%)$ & $19(100)$ & $36(78)$ & $25(78)$ & $21(84)$ \\
\hline & neutral n (\%) & 0 & $10(22)$ & $7(22)$ & $4(16)$ \\
\hline & negative n (\%) & 0 & 0 & 0 & 0 \\
\hline \multirow[t]{6}{*}{ Interprofessional Interaction } & $\mathrm{n}$ & 19 & 47 & 33 & 25 \\
\hline & $M(S D)$ & $\begin{array}{l}28.6 \\
(5.6)\end{array}$ & $\begin{array}{l}28.4 \\
(5.5)\end{array}$ & $30.5(4.4)$ & $27.4(5.5)$ \\
\hline & $\min _{45)}-\max (9-$ & $21-45$ & $15-38$ & $22-44$ & $13-38$ \\
\hline & positive n (\%) & $3(16)$ & $8(17)$ & $1(3)$ & $6(24)$ \\
\hline & neutral n (\%) & $12(63)$ & $25(53)$ & $16(48)$ & $14(56)$ \\
\hline & negative n (\%) & $4(21)$ & $14(30)$ & $16(48)$ & $5(20)$ \\
\hline \multirow[t]{6}{*}{ Interprofessional Relationships } & $\mathrm{n}$ & 20 & 47 & 33 & 25 \\
\hline & $M(S D)$ & $\begin{array}{l}15.6 \\
(4.0)\end{array}$ & $\begin{array}{l}16.2 \\
(3.7)\end{array}$ & $15.8(3.8)$ & $14.8(3.7)$ \\
\hline & $\min _{40)}-\max (8-$ & $8-29$ & $8-26$ & $8-29$ & $8-23$ \\
\hline & positive $\mathrm{n}(\%)$ & $19(95)$ & $42(89)$ & $31(94)$ & $24(96)$ \\
\hline & neutral n (\%) & 0 & $5(11)$ & $1(3)$ & $1(4)$ \\
\hline & negative $\mathrm{n}(\%)$ & $1(5)$ & 0 & $1(3)$ & 0 \\
\hline
\end{tabular}

\section{Discussion}

This study explored factors for implementation of the MCA and attitudes towards interprofessional collaboration of professions in thoracic oncology. In summary, the statements of the staff members show that they see potential in MCA. Especially for patients, some positive benefits for clinical care were evident. Quality of interprofessional collaboration was related to time and personal relationships. 
Implementation required adjustment and adaptation over time which included feeling insecure about responsibilities and role assignment [13]. Physicians and nurse navigators supported each other during the MCs, and the exchange with the wards is also perceived as functioning, especially if a nurse navigator has a good connection to the ward staff. From the wards, an increased transparency regarding the project is desired in order to strengthen the collaboration and the knowledge about the MCA.

Results from interviews and focus group discussions reflected in the attitudes to and experiences with interprofessional work among staff in the oncology department. Participants in the survey appreciated communication and teamwork as well as interprofessional relationships. The assessment of interprofessional interaction was primarily in the neutral range indicating room for improvement. A strong hierarchy among healthcare professionals and widespread stereotypes [14] is not conducive to effective interprofessional interactions. To achieve successful interprofessional interactions, a variety of aspects have to be considered, including equal and collaborative relationships between different professional groups, (un)biased views, open communication, respect and the degradation of hierarchical status [10]. Some of these issues are more difficult to address within interprofessional communication training than others. While openness to communication is a prerequisite for applying training into practice, traditionally hold views on status are less easily overcome [15]. The fact is that this barrier exists in practice and has an influence on an individual's own role within the team which was shown in the interviews. During the implementation of the MCA, physicians and nurses initially struggled to define their roles and responsibilities, but managed to adapt to the challenges of the approach and to strengthen interprofessional interaction over time. For staff members not directly involved in the MCA, it was more difficult to adapt their roles and tasks in relation to the MCA. Future implementation of interprofessional concepts thus need to take into account not only attitudes of individuals but also existing team structures to successfully foster interprofessional interactions.

Further aspects of effective collaboration represent successful communication and teamwork. Communication and teamwork involve exchanging opinions, explaining issues and feeling comfortable working in a group [10]. They are core features of the MCA [16]. For a successful implementation of these aspects, physicians and nurses who put the intervention into practice need to be motivated to adhere to the concept goals and consider themselves an essential part of the implementation [12]. The positive attitude towards communication and teamwork was highlighted in the results of the questionnaire survey and was partly mirrored in the interviews. Nevertheless, interviews showed that physicians in particular did not consider themselves part of the MCA team and referred to "them" whenever current MCA issues were discussed, even when they were involved in MCA discussions with patients. Possible reasons for this are seen in the fact that the "team" did not seem to be defined. Next to the tandem with alternating nurse-physician duos, nurse navigators defined themselves as a team. Beyond the professionals directly involved, the importance of transparency about the concept was underestimated at the beginning of the project, thus hindering the support by the extended healthcare team.

On the other hand, positive experiences with the MCA supported maintenance and further facilitation of already implemented aspects. Even if participants did not receive formal training but were involved in

Page $15 / 19$ 
patient conversations, they observed alleviation through the MCA both for themselves and the patients. The participating healthcare professionals not directly involved in the MCA received additional information about the program from the patients. Positive experiences were reported both by patients and healthcare professionals. Transparency about the approach and involving healthcare professionals not directly conducting patient consultations to keep the whole team informed, foster a community of practice [7] and indirectly strengthen care by giving the patients a generated feeling of team-delivered patient-centred care [17].

Improving patient-centred care is a common goal of interprofessional practice which involves high quality teamwork [18]. Communication is a prerequisite for teamwork. Especially in interprofessionally conducted conversations with patients, participants need to know their own part and the role of the team members. Tasks and responsibilities have to be prepared and coordinated beforehand to achieve successful collaboration with shared accountability [19] without denying individual characteristics of healthcare staff and the team competencies emerging from their interpersonal relationships [20].

Apart from the task of performing trajectory-based conversations in a nurse-physician tandem, prerequisites like sufficient time and space for conducting conversations with the patients as planned posed an additional challenge. Those context factors ("Availability of necessary resource" in the TICD checklist [12]) were not in the focus of this study but emerged as important influences on both fidelity of the intervention and perception of the teamwork. Future implementations should pay stronger attention to context factors (e. g. by using a coding model [21] based on the Consolidated Framework for Implementation Research [22]). Identification of logistic barriers and taking up measures to lower them will not only foster the implementation but also raise the motivation of staff not directly involved in the project, thus strengthening the team and interprofessional interaction [23].

In our study, a change in interprofessional collaboration in the whole department after introducing an interprofessional approach to a small number of healthcare professionals could not be shown during the course of the study. To establish a community of practice in an interprofessional team requires more time for adaptation processes and changes of attitude. Still, our participants reported experiencing advantages of the MCA which further consolidation might be built on.

\section{Conclusions}

In summary, our study showed both positive experiences and potential shortfalls in the implementation of the MCA. Aspects were identified which need a stronger focus in future introduction of trajectory-based conversations in an interprofessional tandem, i.e. preparation for conversations within the tandem and information exchange beyond the tandem to allow patient-centred care by an interprofessional team involving physicians, nurses, therapists, psychosocial professionals and others. Changing roles and responsibilities in the team may initiate a paradigm shift throughout the organisation.

\section{List Of Abbreviations}


MCA Milestone Communication Approach; MCs Milestone Conversations; SD standard deviation; TICD Tailored Intervention in Chronic Diseases; UWE-IP-D German version of the University of the West of England Interprofessional Questionnaire

\section{Declarations}

Ethics approval and consent to participate

The study was approved by the Ethics Committee of the University Hospital Heidelberg, Germany (protocol no. S-561/2017). All methods reported in this paper were performed in accordance with the relevant guidelines and regulations. All participants gave their written informed consent for study participation.

Consent for publication

Not applicable.

Availability of data and materials

The datasets generated and analysed during the current study are not publicly available since participants did not consent to publication. However, anonymized and/or aggregated data are available from the corresponding author on reasonable request.

Competing interests

The authors declare that they have no competing interests.

Funding

This research was funded by the Federal Ministry of Health, Germany (1504-54401), and the National Center for Tumor Diseases (NCT 3.0, G835), Heidelberg, Germany. The funder provided no role in the conception or design of this study, the acquisition, analysis, interpretation of data and drafting of the manuscript.

Authors' contributions

$K K, J B, N D, A S, M V, L H, M T$ and MW conceived and designed the study. JB, SM, AS and CJ acquired the data. KK, JB, SM, ND, LU and AS analysed the data. KK \& JB drafted the manuscript. All authors interpreted the data, critically revised manuscript drafts and read and approved the final manuscript.

Acknowledgements

We would like to gratefully thank all participants for sharing their experiences and perspectives within this study. We sincerely thank Sarah Jane Berger for reviewing language and expression. 


\section{References}

1. Villalobos M: ES05. 02 From Living Longer to Also Living Better-the Role of Communication and Interprofessional Collaboration in Metastatic Lung Cancer. Journal of Thoracic Oncology 2019, 14(10):S24-S25.

2. Feldstain A, Bultz BD, de Groot J, Abdul-Razzak A, Herx L, Galloway L, Chary S, Sinnarajah A: Outcomes from a patient-centered, interprofessional, palliative consult team in oncology. Journal of the National Comprehensive Cancer Network 2018, 16(6):719-726.

3. Homeyer S, Hoffmann W, Hingst P, Oppermann RF, Dreier-Wolfgramm A: Effects of interprofessional education for medical and nursing students: enablers, barriers and expectations for optimizing future interprofessional collaboration-a qualitative study. BMC nursing 2018, 17(1):13.

4. White BAA, Eklund A, McNeal T, Hochhalter A, Arroliga AC: Facilitators and barriers to ad hoc team performance. In: Baylor University Medical Center Proceedings: 2018: Taylor \& Francis; 2018: 380384.

5. Siegle A, Villalobos M, Bossert J, Krug K, Hagelskamp L, Krisam J, Handtke V, Deis N, Junger J, Wensing $\mathrm{M}$ et al: The Heidelberg Milestones Communication Approach (MCA) for patients with prognosis < 12 months: protocol for a mixed-methods study including a randomized controlled trial. Trials 2018, 19(1):438.

6. Krug K, Bossert J, Deis N, Krisam J, Villalobos M, Siegle A, Jung C, Hagelskamp L, Unsold L, Junger J et al: Effects of an Interprofessional Communication Approach on Support Needs, Quality of Life, and Mood of Patients with Advanced Lung Cancer: A Randomized Trial. Oncologist 2021, 26(8):e1445-e1459.

7. Cruess RL, Cruess SR, Steinert Y: Medicine as a Community of Practice: Implications for Medical Education. Academic medicine: journal of the Association of American Medical Colleges 2018, 93(2):185-191.

8. Mahler C, Berger S, Pollard K, Krisam J, Karstens S, Szecsenyi J, Krug K: Translation and psychometric properties of the German version of the University of the West of England Interprofessional Questionnaire (UWE-IP). Journal of interprofessional care 2017, 31(1):105-109.

9. Pollard K, Miers ME, Gilchrist M: Second year scepticism: prequalifying health and social care students' midpoint self-assessment, attitudes and perceptions concerning interprofessional learning and working. Journal of interprofessional care 2005, 19(3):251-268.

10. Pollard KC, Miers ME, Gilchrist M: Collaborative learning for collaborative working? Initial findings from a longitudinal study of health and social care students. Health Soc Care Community 2004, 12(4):346-358.

11. Qualitative content analysis: theoretical foundation, basic procedures and software solution [https://nbn-resolving.org/urn:nbn:de:0168-ssoar-395173]

12. Flottorp SA, Oxman AD, Krause J, Musila NR, Wensing M, Godycki-Cwirko M, Baker R, Eccles MP: A checklist for identifying determinants of practice: a systematic review and synthesis of frameworks 
and taxonomies of factors that prevent or enable improvements in healthcare professional practice. Implementation science: IS 2013, 8:35.

13. May C: Towards a general theory of implementation. Implementation science: IS 2013, 8:18.

14. Kammer JE, Ewers M: Stereotypes of experienced health professionals in an interprofessional context: results from a cross-sectional survey in Germany. Journal of interprofessional care 2021:112.

15. Price S, Doucet S, Hall LM: The historical social positioning of nursing and medicine: implications for career choice, early socialization and interprofessional collaboration. Journal of interprofessional care 2014, 28(2):103-109.

16. Villalobos M, Siegle A, Hagelskamp L, Jung C, Thomas M: Communication along Milestones in Lung Cancer Patients with Advanced Disease. Oncol Res Treat 2019, 42(1-2):41-46.

17. Bilodeau K, Dubois S, Pepin J: Interprofessional patient-centred practice in oncology teams: utopia or reality? Journal of interprofessional care 2015, 29(2):106-112.

18. Bilodeau K, Tremblay D: How oncology teams can be patient-centred? opportunities for theoretical improvement through an empirical examination. Health expectations: an international journal of public participation in health care and health policy 2019, 22(2):235-244.

19. D'Amour D, Oandasan I: Interprofessionality as the field of interprofessional practice and interprofessional education: an emerging concept. Journal of interprofessional care 2005, 19 Suppl 1:8-20.

20. Brashers V, Haizlip J, Owen JA. The ASPIRE Model: Grounding the IPEC core competencies for interprofessional collaborative practice within a foundational framework. J Interprof Care 2020, 34(1):128-132.

21. Rogers L, De Brún A, McAuliffe EJBMRM: Development of an integrative coding framework for evaluating context within implementation science. 2020, 20(1):1-14.

22. Damschroder LJ, Aron DC, Keith RE, Kirsh SR, Alexander JA, Lowery JC: Fostering implementation of health services research findings into practice: a consolidated framework for advancing implementation science. Implementation science 2009, 4(1):1-15.

23. Michie S, van Stralen MM, West R: The behaviour change wheel: A new method for characterising and designing behaviour change interventions. Implementation Science 2011, 6(1):42. 